\title{
Effect of obesity on vedolizumab response in inflammatory bowel disease
}

\author{
Louis J. Levine ${ }^{a}$, Jill K. J. Gaidos ${ }^{\text {b }}$ Deborah D. Proctorb, Artur V. Viana ${ }^{\text {b }}$, Badr AI-Bawardy \\ Yale School of Medicine, New Haven, CT, USA
}

\section{Abstract}

\section{Introduction}

As the global prevalence of obesity in the general population increases, so too does the prevalence of obesity in patients with inflammatory bowel disease (IBD), including ulcerative

${ }^{a}$ Department of Internal Medicine (Louis J. Levine); bection of Digestive Diseases (Jill K. J. Gaidos, Deborah D. Proctor, Artur V. Viana, Badr Al-Bawardy), Yale School of Medicine, New Haven, CT, USA

Conflict of Interest: Dr. Badr Al-Bawardy: Speaker honoraria: AbbVie, Takeda; Dr. Jill K. J. Gaidos: AbbVie Speaker Bureau; All other authors have no disclosures

Correspondence to: Badr Al-Bawardy, MD, Assistant Professor of Medicine, Section of Digestive Diseases, 40 Temple Street, Suite 1A, New Haven, CT, 06510, USA, e-mail: badr.albawardy@yale.edu

Received 11 August 2021; accepted 7 February 2022; published online 21 February 2022

DOI: https://doi.org/10.20524/aog.2022.0699 colitis (UC) and Crohn's disease (CD). The proportion of IBD patients who have obesity, defined as a body mass index (BMI) equal or greater than $30 \mathrm{~kg} / \mathrm{m}^{2}$, reflects the proportion in the general population, which is between $15 \%$ and $40 \%$ based on recent data [1].

The relationship between obesity and IBD outcomes and natural history remains unclear. Retrospective studies have shown that obesity is associated with greater morbidity in IBD patients, including higher rates of hospitalization, rates of colectomy in UC, and disease relapse in CD [2,3]. Conversely, obesity has also been associated with improved outcomes in IBD or a milder phenotype [4]. One large retrospective study found that IBD patients with BMI greater than 30 had fewer hospitalizations $(42.1 \%$ vs. $66.0 \%, \mathrm{P}<0.001)$ and less need for surgery $(41.1 \%$ vs. $61.1 \%, \mathrm{P}=0.02)$ than those with normal BMI [5].

It is important to consider the effect of obesity on weightbased compared to fixed-dose biologics in IBD, as the data are unclear. Pharmacokinetic studies of adalimumab and infliximab in IBD patients with obesity have demonstrated greater drug 
clearance and lower trough concentrations, particularly for adalimumab [6,7]. Another study found that patients with a BMI of $30 \mathrm{~kg} / \mathrm{m}^{2}$ or greater needing dose escalation sooner due to loss of response to adalimumab, but not infliximab, which is dosed based on weight [8]. However, despite infliximab being weight-based, some studies still found a direct correlation between high BMI and treatment failure $[9,10]$. These results imply that obesity may impact the overall drug efficacy.

Vedolizumab (VDZ) is not dosed based on weight. Unfortunately, up to $50 \%$ of IBD patients who initially responded to the standard VDZ treatment may ultimately lose their response to the drug and require dose escalation or drug discontinuation [11]. It is known that VDZ trough drug levels have an inverse relationship to weight [12]. Multiple studies have initially demonstrated an association between higher VDZ trough levels and favorable outcomes [13,14]. However, a recent observational study of pre-dose optimization of VDZ trough levels did not support the notion that higher VDZ trough levels correlate with recapturing response [15]. Further, there are limited data available that show how patients of different BMI respond to the fixed-dose regimen of VDZ. The purpose of this study was to determine the effect of obesity on VDZ response, including the need for dose escalation or discontinuation, as well as the clinical, biochemical and endoscopic response in patients with IBD.

\section{Patients and methods}

This study was a single-center, retrospective chart review of IBD patients seen at the Yale New Haven Health System between $1^{\text {st }}$ July 2014 and $1^{\text {st }}$ September 2020. Inclusion criteria were age $\geq 18$ years, diagnosis of IBD (CD, UC, or indeterminate colitis), and treatment with at least one dose of VDZ during the specified time period. Exclusion criteria were age $<18$, no IBD diagnosis, and no current or prior VDZ use. The study protocol was approved by the Yale University Institutional Review Board (IRB\#2000028937).

Background information, including age, sex, baseline BMI and smoking status, were extracted from each participant's record to form the data set. Disease-related variables included date of IBD diagnosis, IBD subtype, disease location, disease phenotype, and presence of documented extraintestinal manifestations. Treatment-related variables included all current and prior medical and surgical therapies for IBD, specific dates of VDZ usage and dose interval in weeks, use of corticosteroids at time of initiation of VDZ, and use of combination therapy with VDZ, such as a thiopurine or methotrexate. Variables related to disease outcomes included laboratory markers (albumin, C-reactive protein [CRP]) within 30 days prior to starting VDZ and after use, documentation of steroidfree clinical remission (SFCR), and reports from endoscopy, pathology, and radiology pertaining to IBD.

The primary outcome of the study was the need for VDZ dose escalation over time (defined as having the frequency of maintenance VDZ infusions < every 8 weeks) due to partial or total loss of response, or complete discontinuation of the medication during the study time period due to partial or total loss of response. Secondary outcomes were achievement of SFCR, endoscopic remission (defined as absence of ulcers or erosions in CD and Mayo endoscopic score $\leq 1$ for UC), and normal serum CRP (defined as $\leq 5 \mathrm{mg} / \mathrm{L}$ ). Outcomes were compared between patients with $\mathrm{BMI}<30 \mathrm{~kg} / \mathrm{m}^{2}$ or $\geq 30 \mathrm{~kg} / \mathrm{m}^{2}$.

\section{Statistical analysis}

JMP (SAS Institute Inc., Cary, North Carolina, United States) statistical software was used for data analysis. Continuous variables were analyzed using an unpaired Student's $t$-test. Categorical variables were analyzed using Pearson's chi-square test. A time-to-event analysis using a Kaplan-Meier curve was performed and compared between the 2 groups for VDZ treatment persistence. The event was VDZ discontinuation. A log-rank test with a P-value $<0.05$ was considered statistically significant. Multivariate logistic regression models were built to evaluate the association of obesity with VDZ outcomes and the clinically relevant confounders were selected $a$ priori based on univariate analysis findings, clinical experience and the literature. A P-value $<0.05$ was considered statistically significant.

\section{Results}

A total of 190 patients were included in the study (Table 1). The median age was 37 years (range 16-80) and 50.5\% of patients were male. With respect to IBD subtype, $53.7 \%$ of patients had CD (31.7\% with perianal involvement), $42.6 \%$ had UC and $3.7 \%$ had indeterminate colitis. Treatment with combination therapy (a thiopurine or methotrexate) was used in $35.3 \%$ of patients and $52.6 \%$ were on corticosteroids at the time of initiating VDZ. In addition, $28.4 \%$ had prior bowel resection and $78.4 \%$ had prior exposure to biologic agents before starting VDZ. A total of 53 patients had extraintestinal manifestations. Peripheral arthritis was the most common manifestation, present in 47 of these patients. Four patients had erythema nodosum or pyoderma gangrenosum, one patient had both uveitis and erythema nodosum, and one patient had sacroiliitis.

The median BMI was $24.8 \mathrm{~kg} / \mathrm{m}^{2}$ (range 16.2-45.5), with 143 patients $(75.3 \%)$ having a $\mathrm{BMI}<30 \mathrm{~kg} / \mathrm{m}^{2}$ (non-obese). Patients with obesity were more likely to have extraintestinal manifestations compared to patients without obesity $(44.7 \%$ vs. $22.4 \% ; \mathrm{P}=0.003$ ). Otherwise, there were no significant differences between the 2 groups in terms of baseline and disease characteristics (Table 2). Baseline laboratory data within 30 days of starting VDZ were not available for albumin in 99 patients (52\%) and CRP in 140 patients (74\%).

The median follow-up time was 21 months (interquartile range 10.8-40.0). Outcomes were recorded during the most recent follow up noted in the chart. Data regarding SFCR, endoscopic remission and CRP were analyzed within 
Table 1 Baseline characteristics of patients

\begin{tabular}{|c|c|}
\hline Characteristics & Value \\
\hline Median age, years (range) & $37(16-80)$ \\
\hline Male, $\mathrm{n}(\%)$ & $96(50.5)$ \\
\hline $\begin{array}{l}\text { Inflammatory bowel disease subtype } \\
\text { Crohn's disease, } \mathrm{n}(\%) \\
\text { L1 } \\
\text { L2 } \\
\text { L3 } \\
\text { L4 } \\
\text { B1 } \\
\text { B2 } \\
\text { B3 }\end{array}$ & $\begin{array}{c}102(53.7) \\
9(8.8) \\
23(22.5) \\
68(66.7) \\
2(2.0) \\
41(40.2) \\
31(30.4) \\
30(29.4)\end{array}$ \\
\hline Perianal disease, $\mathrm{n}(\%)$ & $31(31.7)$ \\
\hline Ulcerative colitis, n (\%) & $81(42.6)$ \\
\hline Indeterminate colitis, $\mathrm{n}(\%)$ & $7(3.7)$ \\
\hline Proctitis & $1(1.2)$ \\
\hline Left-sided colitis & $29(32.9)$ \\
\hline Pancolitis & $58(65.9)$ \\
\hline Combination therapy, $\mathrm{n}(\%)$ & $67(35.3)$ \\
\hline Thiopurine & $47(70.1)$ \\
\hline Methotrexate & $20(29.9)$ \\
\hline Prior bowel resection, $\mathrm{n}(\%)$ & $54(28.4)$ \\
\hline $\begin{array}{l}\text { Steroid use at time of starting vedolizumab, } \mathrm{n} \\
(\%)\end{array}$ & $100(52.6)$ \\
\hline Previous exposure to biologic agents, $\mathrm{n}(\%)$ & $149(78.4)$ \\
\hline
\end{tabular}

$\leq 6$ months after starting VDZ in the obese group in $6.4 \%$ $(\mathrm{n}=3), 14.9 \%(\mathrm{n}=7)$, and $14.9 \%(\mathrm{n}=7)$, respectively. In the nonobese group, data regarding SFCR, endoscopic remission and CRP were analyzed within $\leq 6$ months after starting VDZ in $20.3 \%(n=29), 18.2 \%(n=26)$, and $18.9 \%(n=27)$, respectively. The rest of the outcomes analyzed were noted $>6$ months after starting VDZ therapy.

The dose of VDZ was escalated in $48.9 \%$ of the obese group compared to $42 \%$ of the non-obese group $(\mathrm{P}=0.4)$ (Fig. 1). VDZ was discontinued in $31.9 \%$ of the obese group versus $53.2 \%$ of the non-obese group $(\mathrm{P}=0.01)$. Vedolizumab treatment persistence at 6 months was $89.3 \%$ in the non-obese group compared to $95.7 \%$ in the obese group. At 12 months, VDZ treatment persistence was $73.6 \%$ in the non-obese group compared to $93.6 \%$ in the obese group (Fig. 2). SFCR was achieved in $57.5 \%$ of the obese group compared to $43.4 \%$ of the non-obese group $(\mathrm{P}=0.22)$. Endoscopic remission was achieved in $60.6 \%$ of the obese group compared to $50.5 \%$ of the non-obese group $(\mathrm{P}=0.32)$. The rate of CRP normalization $(n=145)$ was significantly lower in the obese group compared to the non-obese group ( $46.2 \%$ vs. $66.0 \% ; \mathrm{P}=0.03$ ).

On multivariate analysis, non-obese BMI was significantly associated with VDZ discontinuation (odds ratio [OR] 2.41, 95\% confidence interval $[\mathrm{CI}] 1.11-5.23 ; \mathrm{P}=0.03)$ and normal CRP (OR 2.97, 95\%CI 1.29-6.81; $\mathrm{P}=0.01$ ). In addition, combination therapy with immunomodulators was associated

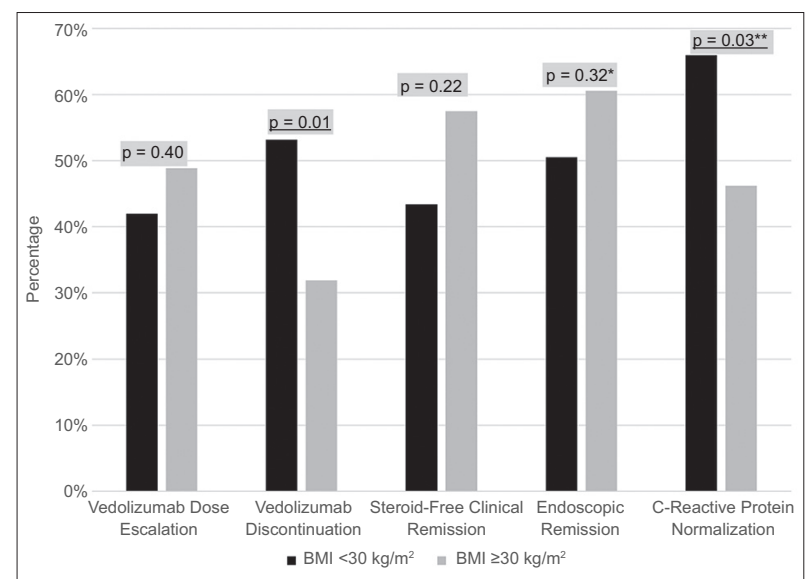

Figure 1 Primary outcomes (VDZ dose escalation and VDZ discontinuation) and secondary outcomes (SFCR, endoscopic remission, CRP normalization) displayed graphically, stratified by $\mathrm{BMI}<30 \mathrm{~kg} / \mathrm{m}^{2}$ or BMI $\geq 30 \mathrm{~kg} / \mathrm{m}^{2}$

${ }^{*}$ Data not available for 62 patients; ${ }^{* *}$ Data not available for 45 patients VDZ, vedolizumab; CRP, C-reactive protein; SFCR, steroid-free clinical remission; $B M I$, body mass index

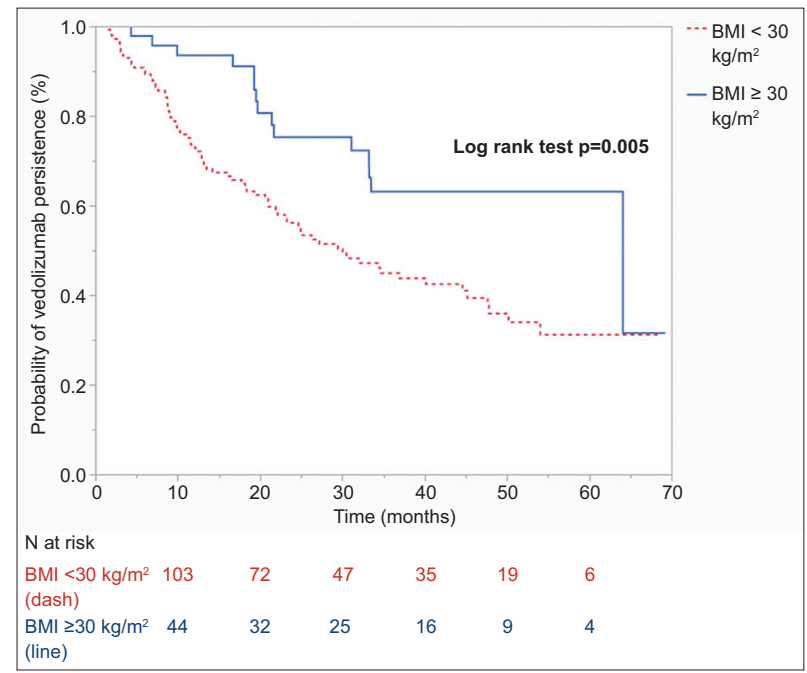

Figure 2 Kaplan-Meier time-to-event analysis of vedolizumab treatment persistence between inflammatory bowel disease patients with BMI $<30 \mathrm{~kg} / \mathrm{m}^{2}$ (dash) and BMI $\geq 30 \mathrm{~kg} / \mathrm{m}^{2}$ (line)

$B M I$, body mass index

with VDZ dose escalation (OR 2.39, 95\%CI 1.23-4.68; $\mathrm{P}=0.01$ ), and VDZ discontinuation (OR 2.013, 95\%CI 1.02-3.96; $\mathrm{P}=0.043)$.

\section{Discussion}

In this single-center retrospective study, which included 190 IBD patients with and without obesity, we found that almost half of all patients required dose escalation of VDZ, consistent with other studies [11]. We also found that, compared to non-obese BMI, obesity was not associated with greater 
Table 2 Patient characteristics while on vedolizumab, stratified by BMI $<30 \mathrm{~kg} / \mathrm{m}^{2}$ or $\geq 30 \mathrm{~kg} / \mathrm{m}^{2}$

\begin{tabular}{|c|c|c|c|}
\hline Patient characteristics & $\begin{array}{l}\mathrm{BMI}<30(\mathrm{n}=143), \\
\mathrm{n}(\%), \text { mean }(\mathrm{SD})\end{array}$ & $\begin{array}{l}\mathrm{BMI} \geq 30(\mathrm{n}=47) \\
\mathrm{n}(\%), \text { mean }(\mathrm{SD})\end{array}$ & P-value \\
\hline \multicolumn{4}{|l|}{ Baseline characteristics } \\
\hline Age (years) & $40.7(16.6)$ & $43.7(14.9)$ & 0.24 \\
\hline Female sex & $69(48.3)$ & $25(53.2)$ & 0.56 \\
\hline Disease duration (years) & $11.5(10.5)$ & $12(8.1)$ & 0.72 \\
\hline $\begin{array}{l}\text { Albumin at start of } \\
\text { vedolizumab* }\end{array}$ & $3.9(0.55)$ & $4.1(0.41)$ & 0.07 \\
\hline $\begin{array}{l}\text { C-reactive protein at start of } \\
\text { vedolizumab }(\mathrm{mg} / \mathrm{L}) \dagger\end{array}$ & $12.8(23.2)$ & $13.4(32.9)$ & 0.94 \\
\hline \multicolumn{4}{|l|}{ Disease characteristics } \\
\hline \multicolumn{4}{|l|}{ Disease type } \\
\hline Crohn's disease & $77(53.9)$ & $25(53.2)$ & 0.79 \\
\hline Ulcerative colitis & $60(41.9)$ & $21(44.7)$ & \\
\hline Indeterminate colitis & $6(4.2)$ & $1(2.1)$ & \\
\hline Prior bowel resection & $43(30.1)$ & $11(23.4)$ & 0.38 \\
\hline $\begin{array}{l}\text { Presence of extraintestinal } \\
\text { manifestations }\end{array}$ & $32(22.4)$ & $21(44.7)$ & 0.003 \\
\hline \multicolumn{4}{|l|}{ Medication factors } \\
\hline Combination therapy use & $48(33.6)$ & $19(40.4)$ & 0.39 \\
\hline Prior biologic agent use & $111(77.6)$ & $38(80.8)$ & 0.64 \\
\hline Steroid use at vedolizumab induction & $79(55.2)$ & $21(44.7)$ & 0.23 \\
\hline
\end{tabular}

rates of VDZ dose escalation and that the rates of SFCR and endoscopic remission were not significantly different between the 2 groups. VDZ discontinuation rates were lower in the obese compared to the non-obese group. This was supported by a time-to-event analysis for VDZ persistence. However, the data did show that the rate of CRP normalization after treatment with VDZ was significantly higher in the non-obese group of IBD patients.

The pharmacology of the early monoclonal antibodies approved for IBD, such as infliximab, adalimumab and certolizumab, has been studied extensively [16]. Studies evaluated the impact of patient-related factors, including body weight, on the pharmacokinetics of monoclonal antibodies. Increased body weight is associated with an increased volume of distribution of monoclonal antibodies [17]. Because of the impact of obesity on pharmacokinetic parameters, infliximab is dosed based on body weight. However, the relationship between body weight and drug clearance of monoclonal antibodies is not linear, suggesting that there are other factors besides weight that also impact drug clearance and ultimately drug levels [16]. These findings make therapeutic drug monitoring crucial, particularly in patients with obesity or with monoclonal antibodies that are not based on weight.

Specific pharmacologic parameters of VDZ have also been studied [18]. During its clinical trials, VDZ was first administered as a weight-based dose, like prior monoclonal antibodies. However, further investigation found that weight-adjusted dosing did not have a significant advantage over fixed dosing. Except for patients with weight $>120 \mathrm{~kg}$, one study found no clinically meaningful difference in the clearance of the fixed dose of VDZ based on weight [19]. This led to FDA approval for a fixed dose of $300 \mathrm{mg}$ intravenously every 8 weeks.

Still, there is a paucity of current data on the effect of obesity on actual clinical response to VDZ in IBD patients. Existing literature has investigated the effect of obesity on clinical response to other biologics in other autoimmune conditions [20]. A 2018 study by Singh et al found that, for autoimmune diseases other than IBD, higher BMI is associated with a worse clinical response to tumor necrosis factor (TNF)- $\alpha$ inhibitors. For example, one observational study found that patients with rheumatoid arthritis and obesity treated with the weight-based dosing of infliximab had a lesser reduction in disease severity scores than those without obesity [21]. This relationship was not present for patients with IBD, however. A pooled analysis of over 1200 IBD patients found that obesity was not associated with an inferior response to infliximab (based on CD Activity Index scores and endoscopic remission), regardless of IBD subtype [22]. As in this latter analysis of infliximab in IBD, our study with VDZ did not find a significant impact of obesity on SFCR or endoscopic remission in IBD patients.

Last, we found that CRP normalization occurred more frequently in IBD patients with BMI $<30 \mathrm{~kg} / \mathrm{m}^{2}$ than in those with BMI $\geq 30 \mathrm{~kg} / \mathrm{m}^{2}$. However, the rates of endoscopic remission were similar between the 2 groups. This finding supports existing studies that cite obesity as a persistent state of chronic inflammation [23]. CRP is a non-specific inflammatory marker often elevated in patients with obesity. This may explain why IBD patients with obesity treated with VDZ may have had less of a decline in their CRP after treatment. 
Overall, this study offers a median extended follow-up time of almost 2 years, longer than some existing studies evaluating the effect of obesity on the response to monoclonal antibodies in IBD [22]. Additionally, existing studies have focused solely on TNF- $\alpha$ inhibitors, whereas this study assessed the response to VDZ. This study also offers an analysis of a control group with similar baseline characteristics.

There are some limitations to our study. First, there are inherent limitations of a single-center retrospective study. Given the nature of retrospective reviews, there are patients lost to follow up or with missing data, as discussed in the Results section. Second, despite controlling for variables associated with disease activity and severity, potential unaccounted confounders could still exist. Third, although BMI is used widely, it does have limitations, including the inability to differentiate visceral adiposity from lean body mass. Visceral adiposity may be a more reflective indicator that could potentially play a role in evaluating the response to biologics in IBD, although current data are conflicting [24,25].

In conclusion, this study found that obesity as measured by BMI was not associated with an increased need for dose escalation of VDZ in patients with IBD. This supports the current fixed-dose regimen of VDZ for these patients. Future

\section{Summary Box}

\section{What is already known:}

- Vedolizumab (VDZ) is a monoclonal antibody approved for inflammatory bowel disease (IBD), administered as a non-weight-based, fixed dose

- Higher body mass index (BMI) is associated with lower serum VDZ levels

- Up to half of IBD patients who initially responded to VDZ may lose their response and require dose escalation or drug discontinuation

\section{What the new findings are:}

- Higher BMI was not associated with higher rates of VDZ dose escalation

- Rates of steroid-free clinical remission and endoscopic remission were not significantly different for patients with increased BMI

- Higher BMI was associated with lower rates of VDZ discontinuation and C-reactive protein normalization

large, prospective studies, utilizing other markers such as visceral adiposity, may better identify the patterns and mechanisms of how obesity affects the response to biologics in IBD.

\section{References}

1. Singh S, Dulai PS, Zarrinpar A, Ramamoorthy S, Sandborn WJ. Obesity in IBD: epidemiology, pathogenesis, disease course and treatment outcomes. Nat Rev Gastroenterol Hepatol 2017; 14:110-121.

2. Jain A, Nguyen NH, Proudfoot JA, et al. Impact of obesity on disease activity and patient-reported outcomes measurement information system (PROMIS) in inflammatory bowel diseases. Am J Gastroenterol 2019;114:630-639.

3. Seminerio JL, Koutroubakis IE, Ramos-Rivers C, et al. Impact of obesity on the management and clinical course of patients with inflammatory bowel disease. Inflamm Bowel Dis 2015;21:2857-2863.

4. Pringle PL, Stewart KO, Peloquin JM, et al. Body mass index, genetic susceptibility, and risk of complications among individuals with Crohn's disease. Inflamm Bowel Dis 2015;21:2304-2310.

5. Flores A, Burstein E, Cipher DJ, Feagins LA. Obesity in inflammatory bowel disease: a marker of less severe disease. Dig Dis Sci 2015;60:2436-2445.

6. Sharma S, Eckert D, Hyams JS, et al. Pharmacokinetics and exposure-efficacy relationship of adalimumab in pediatric patients with moderate to severe Crohn's disease: results from a randomized, multicenter, phase-3 study. Inflamm Bowel Dis 2015;21:783-792.

7. Dotan I, Ron Y, Yanai $\mathrm{H}$, et al. Patient factors that increase infliximab clearance and shorten half-life in inflammatory bowel disease: a population pharmacokinetic study. Inflamm Bowel Dis 2014;20:2247-2259.

8. Bhalme M, Sharma A, Keld R, Willert R, Campbell S. Does weight-adjusted anti-tumour necrosis factor treatment favour obese patients with Crohn's disease? Eur J Gastroenterol Hepatol 2013;25:543-549.

9. Kurnool S, Nguyen NH, Proudfoot J, et al. High body mass index is associated with increased risk of treatment failure and surgery in biologic-treated patients with ulcerative colitis. Aliment Pharmacol Ther 2018;47:1472-1479.

10. Harper JW, Sinanan MN, Zisman TL. Increased body mass index is associated with earlier time to loss of response to infliximab in patients with inflammatory bowel disease. Inflamm Bowel Dis 2013;19:2118-2124.

11. Peyrin-Biroulet L, Danese S, Argollo M, et al. Loss of response to vedolizumab and ability of dose intensification to restore response in patients with Crohn's disease or ulcerative colitis: a systematic review and meta-analysis. Clin Gastroenterol Hepatol 2019;17:838-846.

12. Al-Bawardy B, Ramos GP, Willrich MAV, et al. Vedolizumab drug level correlation with clinical remission, biomarker normalization, and mucosal healing in inflammatory bowel disease. Inflamm Bowel Dis 2019;25:580-586.

13. Ungar B, Kopylov U, Yavzori M, et al. Association of vedolizumab level, anti-drug antibodies, and $\alpha 4 \beta 7$ occupancy with response in patients with inflammatory bowel diseases. Clin Gastroenterol Hepatol 2018;16:697-705.

14. Vaughn BP, Yarur AJ, Graziano E, et al. Vedolizumab serum trough concentrations and response to dose escalation in inflammatory bowel disease. J Clin Med 2020;9:3142.

15. Ungar B, Malickova K, Hanžel J, et al. Dose optimisation for loss of response to vedolizumab- pharmacokinetics and immune mechanisms. J Crohns Colitis 2021;15:1707-1719.

16. Ordás I, Mould DR, Feagan BG, Sandborn WJ. Anti-TNF monoclonal antibodies in inflammatory bowel disease: pharmacokinetics-based dosing paradigms. Clin Pharmacol Ther 2012;91:635-646.

17. Ternant D, Aubourg A, Magdelaine-Beuzelin C, et al. Infliximab 
pharmacokinetics in inflammatory bowel disease patients. Ther Drug Monit 2008;30:523-529.

18. Rosario M, Dirks NL, Milch C, et al. A review of the clinical pharmacokinetics, pharmacodynamics, and immunogenicity of vedolizumab. Clin Pharmacokinet 2017;56:1287-1301.

19. Rosario M, Dirks NL, Gastonguay MR, et al. Population pharmacokinetics-pharmacodynamics of vedolizumab in patients with ulcerative colitis and Crohn's disease. Aliment Pharmacol Ther 2015;42:188-202.

20. Singh S, Facciorusso A, Singh AG, et al. Obesity and response to anti-tumor necrosis factor- $\alpha$ agents in patients with select immune-mediated inflammatory diseases: A systematic review and meta-analysis. PLoS One 2018;13:e0195123.

21. Klaasen R, Wijbrandts CA, Gerlag DM, Tak PP. Body mass index and clinical response to infliximab in rheumatoid arthritis.
Arthritis Rheum 2011;63:359-364.

22. Singh S, Proudfoot J, Xu R, Sandborn WJ. Obesity and response to infliximab in patients with inflammatory bowel diseases: pooled analysis of individual participant data from clinical trials. Am J Gastroenterol 2018;113:883-889.

23. Choi J, Joseph L, Pilote L. Obesity and C-reactive protein in various populations: a systematic review and meta-analysis. Obes Rev 2013;14:232-244.

24. Bryant RV, Schultz CG, Ooi S, et al. Visceral adipose tissue is associated with stricturing Crohn's disease behavior, fecal calprotectin, and quality of life. Inflamm Bowel Dis 2019;25:592-600.

25. Rowan CR, McManus J, Boland K, O'Toole A. Visceral adiposity and inflammatory bowel disease. Int $J$ Colorectal Dis 2021;36:2305-2319. 